\title{
A Comparative Study of Water Lubricated Journal Bearings with Rotating and Stationary Recesses for Fuel Cell Compressor
}

\author{
Tianming Ren ${ }^{\mathrm{a}}$, Ming Feng ${ }^{\mathrm{b}}$ \\ University of Science and Technology Beijing, School of Mechanical Engineering, Beijing 100083, \\ China \\ bray_1107@163.com, ${ }^{\mathrm{b}}$ mingfeng@me.ustb.edu.cn
}

\begin{abstract}
Keywords: water-lubricated bearing; stability; turbulence; recess location; fuel cell
Abstract. This paper presents a stability investigation of the water-lubricated journal bearings with rotating or stationary recesses. The dynamic coefficients and stability threshold speed of the bearings are numerically calculated by solving the turbulent Reynolds equation by using FEM. The theoretical results show that the bearing with rotating recesses possesses periodical dynamic coefficients and stability threshold speed whose frequency is equal to the product of the recess number by the rotation frequency. The rotating of the recess decreases the bearing stability, especially at low rotating speed. In addition, the recesses number has different influence on the stability of the two bearings. The validity of the theoretical analysis was verified by the experiments on the built compressor.
\end{abstract}

\section{Introduction}

Fuel cell vehicles have achieved an impressive development in these years. As the core component in the vehicle, an air compressor is needed to supply pressured oxygen to the stack. Centrifugal type compressor is regarded to be an ideal solution for its high efficiency and compact structure. Fluid film journal bearings are recommended in the high speed machine for their low noise and long service life. The authors have already showed the possibility and merits of using water-lubricated bearing in the fuel cell compressor [1]. However, the inherent cross-coupling stiffness coefficients of fluid film journal bearings can lead to whirl motion, which threatens the stability of the rotor-bearing system.

Many bearing configurations have been proposed to improve the stability, recessed bearings are mostly recommended for their excellent overall performance. The recessed bearings can be classified as bearing with rotating recesses (BR) and bearing with stationary recesses (BS). Bonneau and Absi [2] pointed out that BR produces cyclic load with a frequency equal to the product of the groove number by the frequency of rotation of the shaft. However, their investigations only studied the aerodynamic journal bearing with herringbone grooves under laminar condition. The high rotating speed and the low viscosity of water make the lubrication film flows turbulently. The effect of turbulence should also be considered in the analysis. Hashimoto [3] analyzed the dynamic characteristics of the water lubricated plain journal bearings with considering turbulent effect. They found that the bearing stability is overestimated if the influence of flow regime is neglected. However, the effects of stationary or rotating recesses were not considered with.

This paper analyzes the dynamic characteristics of both BR and BS with a circumferential central supply groove. The dynamic coefficients and stability threshold speed (STS) of the bearings are obtained with considering the influence of turbulence. The effects of the rotating and stationary recesses on the bearing stability are investigated.

\section{Bearing Model and Governing Equations}

The water lubricated recessed journal bearings are shown in Fig.1. Both of the two bearings have shallow recesses and a circumferential central groove for water supply. However, one has recessed journal with plain sleeve (Fig. 1(a)) while the other one has plain journal with recessed sleeve (Fig. 1(b)). In the BS, there are deeper recesses at the upstream to help eliminate cavitation phenomenon. 

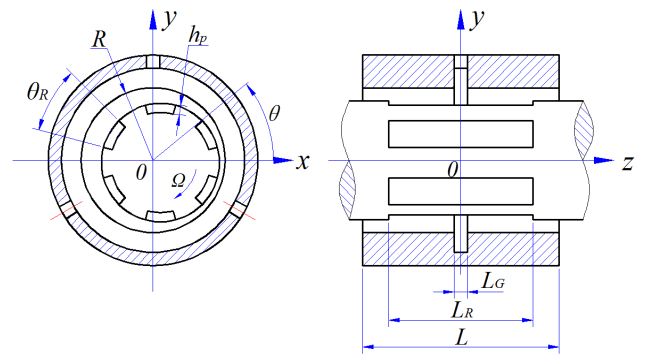

(a) BR
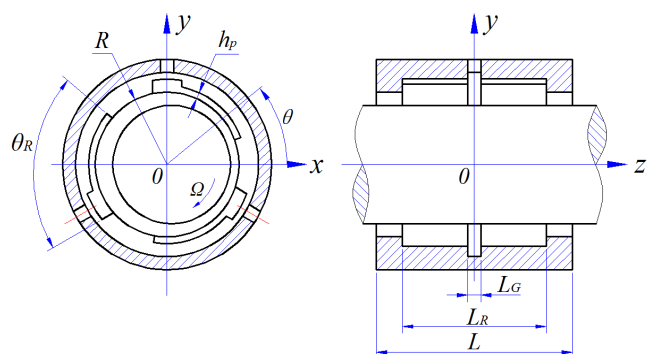

(b) BS

Fig. 1 Schematic diagram of recessed journal bearings

$R$ is the bearing radius, $L$ is the bearing axial width, $h_{p}$ is the recess depth, $L_{R}$ and $\theta_{R}$ are the recess axial and circumferential width, respectively. $L_{G}$ is the axial width of the supply groove, $\delta=N \theta_{R} / 2 \pi$ represents the dimensionless recess circumferential width, where $N$ is the recess number. $\Omega$ is the rotating speed. The main geometrical parameters of the two bearings are given in Tab. 1.

Tab. 1 Main bearing geometrical parameters

\begin{tabular}{c|cc}
\hline & BR & BS \\
\hline Bearing radius $R / m m$ & 15 & 15 \\
Bearing width $L / m m$ & 17 & 17 \\
Bearing clearance $h_{0} / m m$ & 0.02 & 0.02 \\
Recess depth $h_{p} / m m$ & 0.02 & 0.02 \\
Recess axial width $L_{R} / m m$ & 13 & 13 \\
Recess circumferential width $\delta$ & 0.5 & 0.6 \\
Rececess number $N$ & $3,4,5,6$ & $3,4,5,6$ \\
Supply groove width $L_{G} / m m$ & 1 & 1 \\
\hline
\end{tabular}

The modified Reynolds equation with considering the influence of turbulence is

$$
\begin{gathered}
\frac{\partial}{R \partial \theta}\left(\frac{h^{3}}{G_{\theta} \mu} \frac{\partial P}{R \partial \theta}\right)+\frac{\partial}{\partial z}\left(\frac{h^{3}}{G_{z} \mu} \frac{\partial P}{\partial z}\right)=\frac{U}{2} \frac{\partial h}{R \partial \theta}+\frac{\partial h}{\partial t} \\
G_{\theta}=12+0.0136 \mathrm{Re}^{0.9} \\
G_{z}=12+0.0043 \mathrm{Re}^{0.98} \\
h=h_{0}+\Delta h_{p}+e_{x} \cos \theta+e_{y} \sin \theta
\end{gathered}
$$

where $G_{\theta}$ and $G_{z}$ are turbulence coefficients given by $\mathrm{Ng}$ and $\mathrm{Pan}$ [4]. For laminar flow conditions, $G_{\theta}$ $=G_{z}=12 . P$ is the film pressure, $\mu$ is the water dynamic viscosity, $U$ is the bearing rotating velocity, $\theta$ and $z$ are circumferential and axial coordinate respectively, $R e$ is the Reynolds number, $h_{0}$ is the bearing clearance. For the film in the recess, $\Delta=1 ; \Delta=0$ for the else. $e_{x}$ and $e_{y}$ are the eccentricity along $x$ and $y$ direction, respectively.

The dimensionless stiffness and damping coefficients, $\bar{k}_{i j}$ and $\bar{c}_{i j}(\mathrm{i}, \mathrm{j}=\mathrm{x}, \mathrm{y})$, are defined as

$$
\bar{k}_{i j}=K_{i j}\left(h_{0} / R\right)^{3} / \mu L \Omega, \bar{c}_{i j}=C_{i j}\left(h_{0} / R\right)^{3} / \mu L
$$

To a rigid Jeffcott rotor supported horizontally by two identical journal bearings, the STS is

$$
\begin{gathered}
\omega_{s}=\sqrt{k_{e q} / \gamma_{s}^{2}} \\
k_{e q}=\left(\bar{c}_{x x} \bar{k}_{y y}+\bar{c}_{y y} \bar{k}_{x x}-\bar{c}_{x y} \bar{k}_{y x}-\bar{c}_{y x} \bar{k}_{x y}\right) /\left(\bar{c}_{x x}+\bar{c}_{y y}\right) \\
\gamma_{s}^{2}=\left[\left(k_{e q}-\bar{k}_{x x}\right)\left(k_{e q}-\bar{k}_{y y}\right)-\bar{k}_{x y} \bar{k}_{y x}\right] /\left(\bar{c}_{x x} \bar{c}_{y y}-\bar{c}_{x y} \bar{c}_{y x}\right)
\end{gathered}
$$

where $k_{e q}$ and $\gamma_{s}$ are the equivalent stiffness and whirl-frequency ratio, respectively. 


\section{Theoretical Analysis}

Based on the finite element method, Eq. (1) is solved. The STS is then obtained by Eqs. (6)-(8). The theoretical analysis were taken at the rotating speed of $80000 \mathrm{rpm}$ with an eccentricity of $e_{x}=0.3, e_{y}=$ 0. Fig. 2 shows the dynamic coefficients and STS of the BR with different recess numbers versus rotating time. It can be seen clearly that the dynamic coefficients and the STS of BR are all periodical functions whose amplitudes decrease with the increasing of recess number. In one revolution $(0.75 \mathrm{~ms})$, the periodicity number is exactly equal to the recess number. So the frequencies of dynamic coefficients and STS of BR are equal to the product of the recess number by the rotation frequency. However, the period was not found in the characteristics of BS.

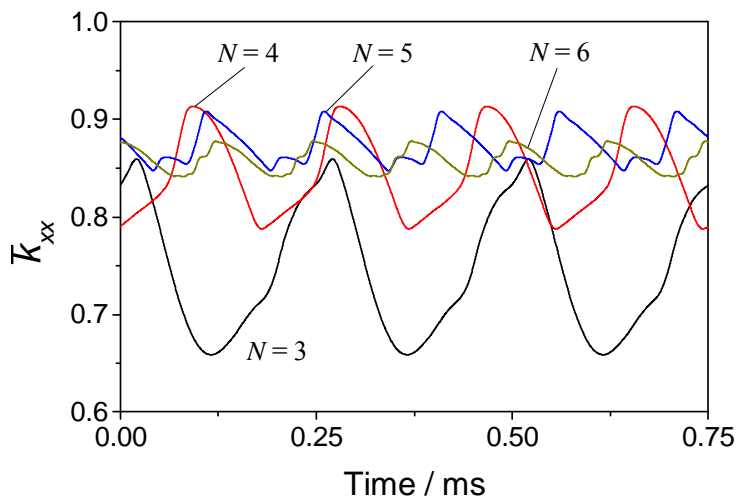

(a) $\bar{k}_{x x}$ of the BR

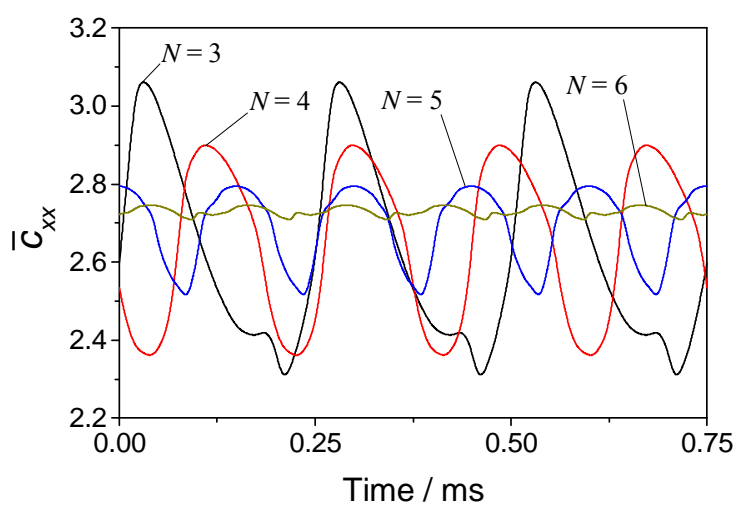

(a) $\bar{c}_{x x}$ of the BR

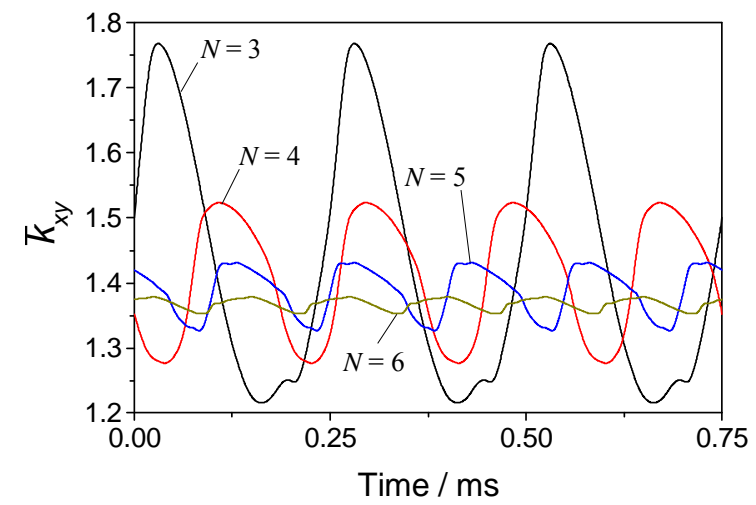

(b) $\bar{k}_{x y}$ of BR

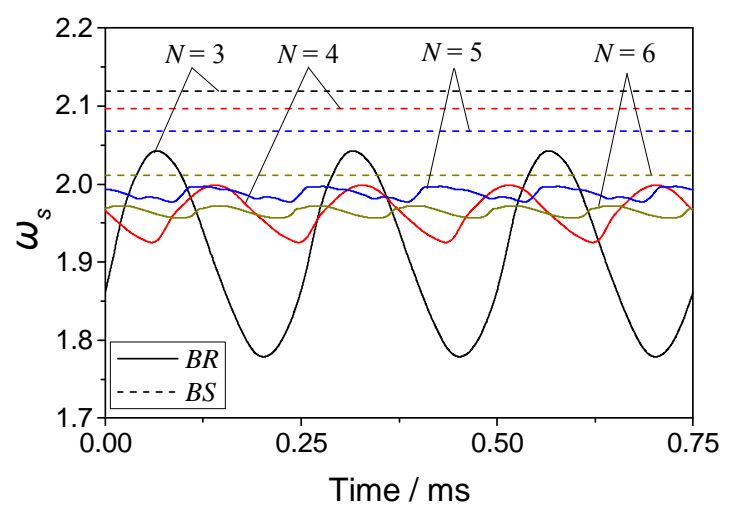

(d) $\omega$ s of BR and BS

Fig. 2 Dynamic coefficients and STS of the bearing versus rotating time

The STS of the BR and BS are compared in Fig. 2(d). The recess number shows different influence on the two bearings. The increase of recess number increases the stability of BR, while decreases that of BS. So the optimal recess numbers are 6 and 3 for BR and BS, respectively. However, the BS still has higher STS than BR at all the calculated recess numbers.

\section{Experimental Verification}

To verify the theoretical results, a motorized centrifugal compressor with the water lubricated bearings was built up [1]. The bearing vibration data were collected by the transducer horizontally fixed at the front and rear bearing positions, as seen in Fig.3. BR and BS with the optimal recess number were applied successively in the compressor.

Fig. 4 shows the STS of the two bearings versus rotating speed $\left(e_{x}=0.3, e_{y}=0\right)$. BS has higher STS than BR at the whole speed range, especially at low rotating speed. This indicates that the rotating of the recess decreases the bearing stability substantially at low rotating speed. However, the rotating effect weakens with the increase of the rotating speed. 




Fig. 3 Test compressor for the two bearings

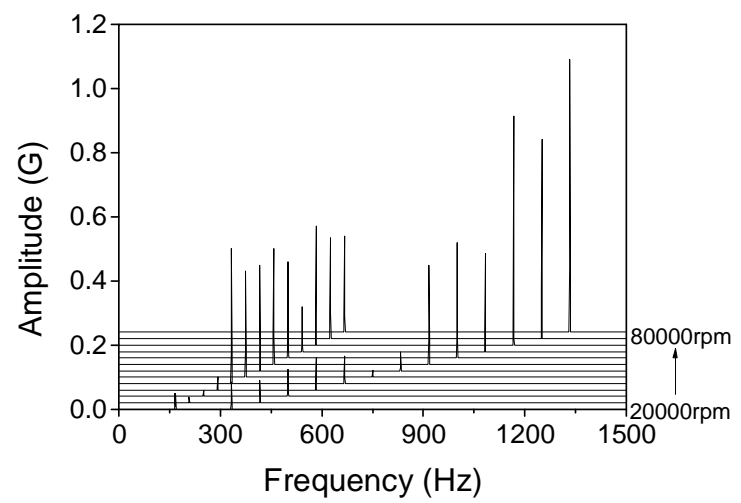

(a) BR

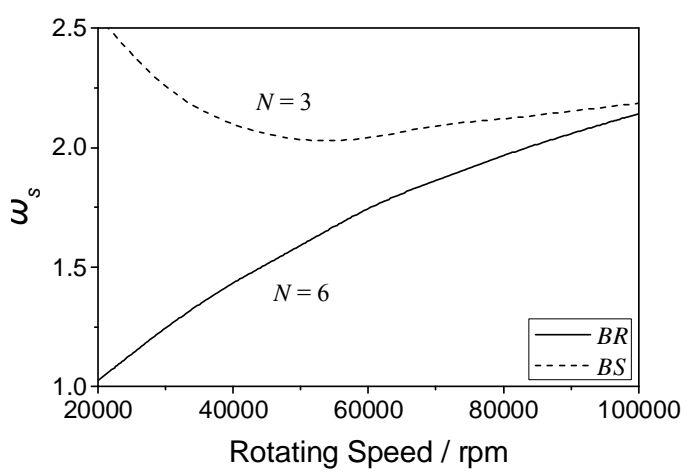

Fig. 4 STS versus rotating speed



(b) BS

Fig. 5 Waterfall Plot of Bearing Vibration

Fig. 5 shows the experimental waterfalls of the two bearings. With the BR, the half whirl was observed at the whole experimental speed range. Meanwhile, the half whirl of BS does not appear until the speed of $55000 \mathrm{rpm}$. This indicates that the water film of BR was instable from the very beginning, the rotating of the recess decreases the bearing stability. As for the high rotating speed, both of the two bearings were instable, the rotating effect of the recess was not obvious. The experiments were in good agreement with the theoretical prediction.

\section{Summary}

The stability of two water lubricated bearings was theoretically and experimentally analyzed. The BS possesses periodical dynamic coefficients and STS whose frequency is equal to the product of the recess number by the rotation frequency. The rotating of the recess decreases the bearing stability, especially at low rotating speed. The recess number has different influence on the two bearings. The increase of recess number increases the stability of BR, while decreases that of the BS. The validity of the theoretical analysis was verified by the experiments.

\section{References}

[1] Ming Feng, Tianming Ren, Application of water-lubricated bearings in motorized centrifugal air compressor for fuel cell automotive, Applied Mechanics and Materials, 607-671(2014), pp.920-923.

[2] Bonneau, D., and Absi, J., Analysis of Aerodynamic Journal Bearings With Small Number of Herringbone Grooves by Finite Element Method, ASME J. Tribol., 116(1994), pp. 698-704.

[3] Hashimoto, H., Wada, S. and Ito, J., An Application of Short Bearing Theory to Dynamic Characteristics Problems of Turbulent Journal Bearings, ASME J. Tribol., 109(1987), pp.307-314.

[4] C. W. Ng, and C. H. T. Pan, A Linearized Turbulent Lubrication Theory, Trans. ASME, Ser. D, 87(1965), pp.675-688. 